\title{
Supporting Information: Origin of Surface-Driven Passive Liquid Flows
}

\author{
Sumith YD and Shalabh C. Maroo*
}

Department of Mechanical and Aerospace Engineering, Syracuse University, Syracuse, New York 13244, United States

\section{PRESSURE ESTIMATION}

Block-diagram of the simulation domain is shown in Fig. S1. The pressure has 9 tensor components, out of which 3 are of primary interest in MD simulations, tensors in planes $\mathrm{XX}, \mathrm{YY}$ and $\mathrm{ZZ}$ named as $P_{X X}, P_{Y Y}$ and $P_{Z Z}$.

The normal pressure is defined as $P_{N}=P_{Z Z}$, tangential pressure as $P_{T}=\frac{P_{X X}+P_{Y Y}}{2}$

The surface tension is estimated as $\gamma=\int\left(P_{N}-P_{T}\right) d z$

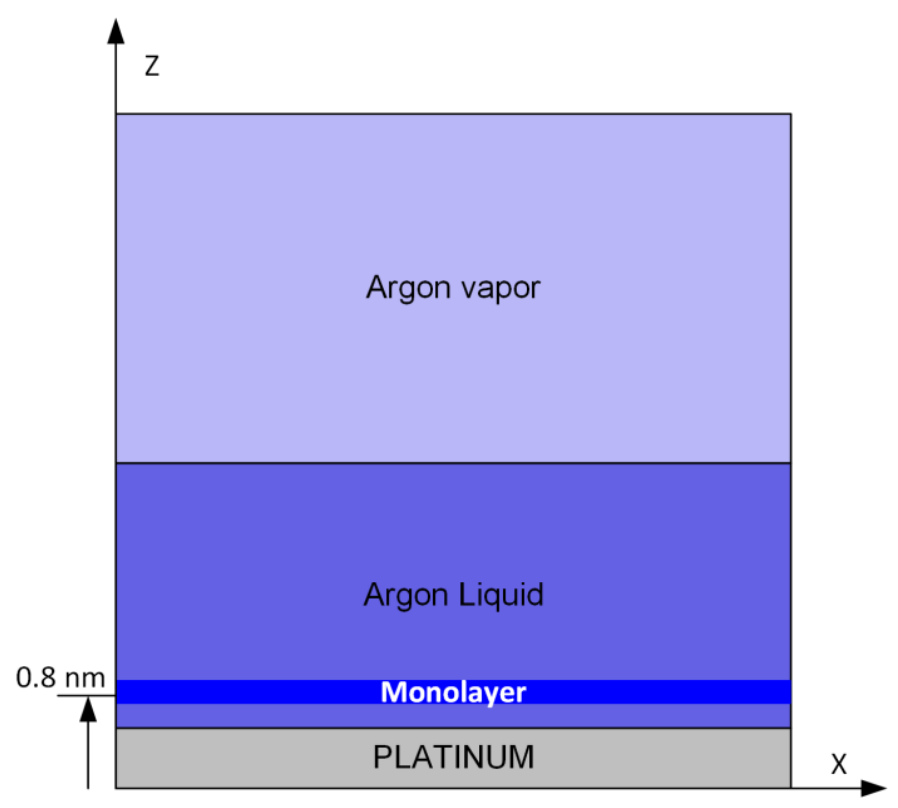

Figure S1. Representation of monolayer above the platinum surface. The lateral direction is X-axis and vertical direction is the Z-axis. 


\section{MONOLAYER CHARACTERIZATION}

Pressure, density, temperature and surface tension is studied in detail here. Figure S2 and Figure $\mathrm{S} 3$ is derived from the equilibrium simulations of argon film on top of platinum. Figure S4 is the detailed analysis for the passive flow case when the mid temperature was $145 \mathrm{~K}$ and sides were at $90 \mathrm{~K}$.

(a)

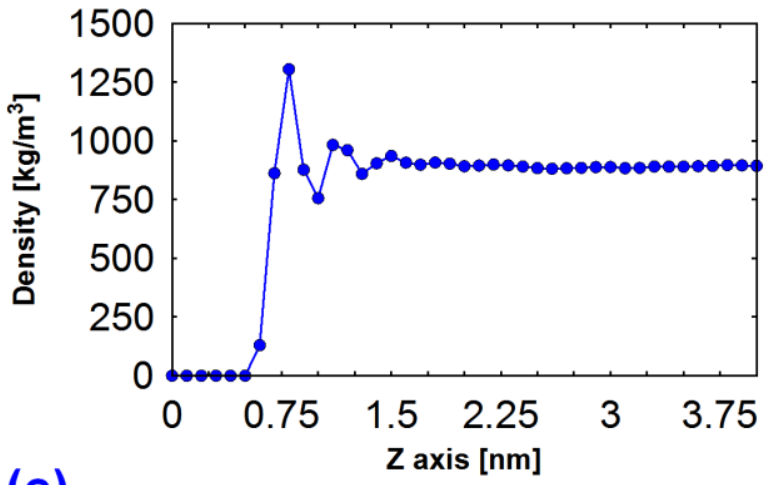

(c)

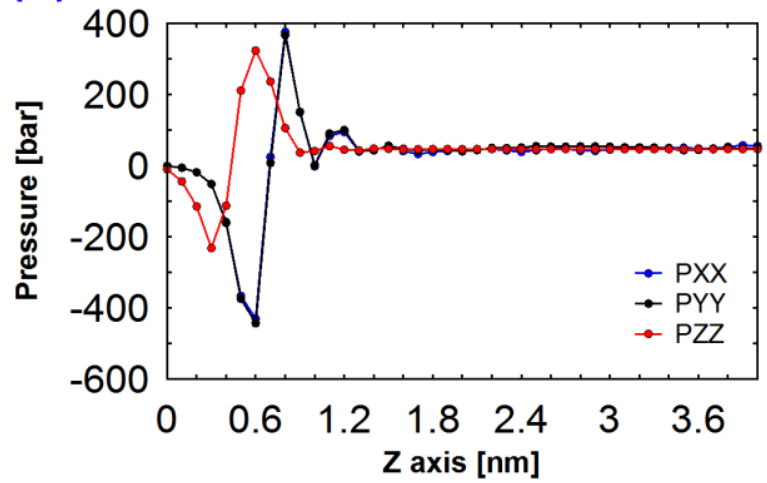

(b)

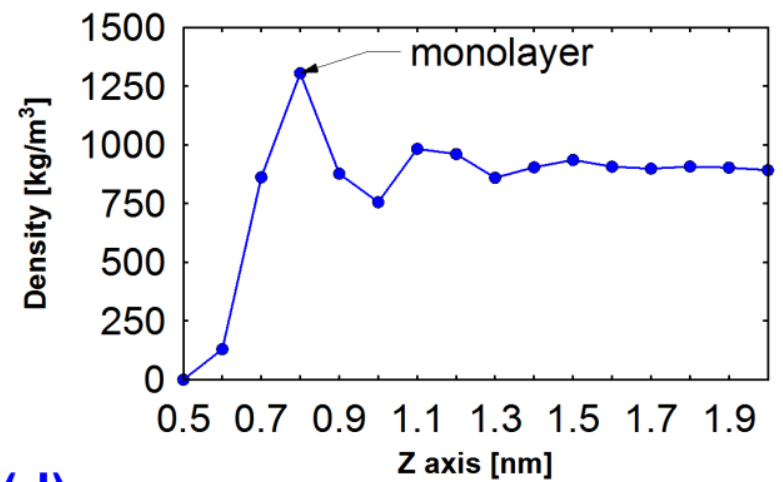

(d)

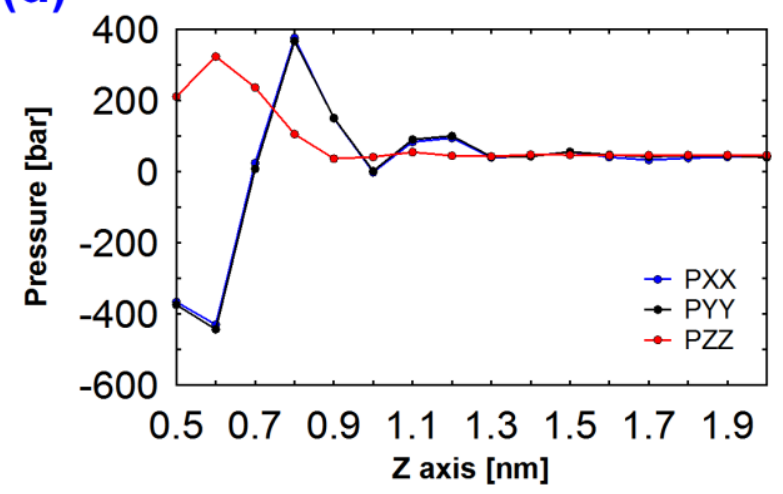

Figure S2. Density and pressure variations of argon above the platinum surface for surface temperature of 145 K. (a) Density variation along Z-axis (normal to platinum surface). (b) Close-up view of density near the surface, formation of the monolayer at $0.8 \mathrm{~nm}$ away from origin is shown. (c) Behavior of pressure tensor components along $\mathrm{X}, \mathrm{Y}$, and $\mathrm{Z}$ axes are shown up to $4 \mathrm{~nm}$. (d) Zoomed in view of the pressure components which show a strong negative pressure existence near surface. 

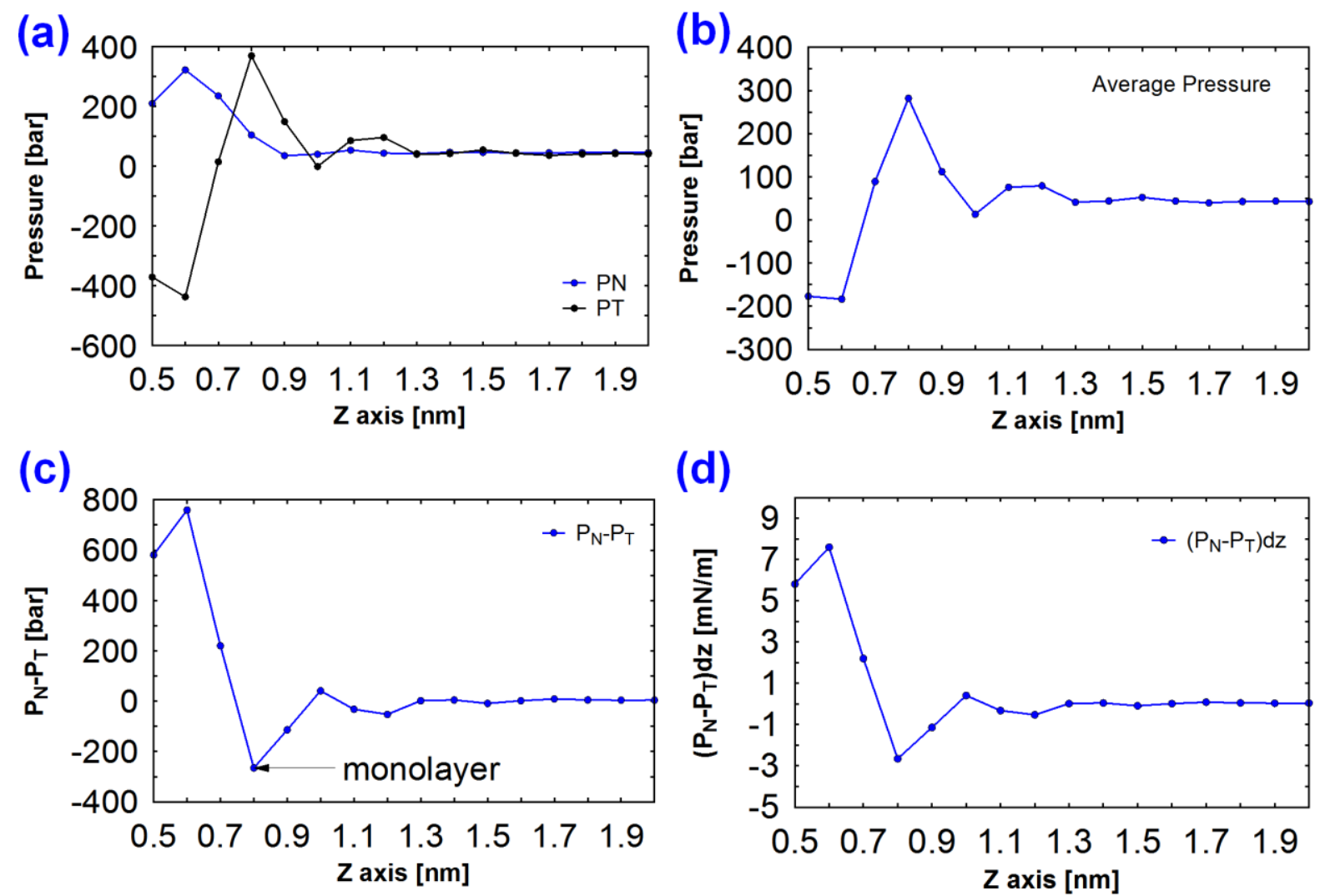

(d)

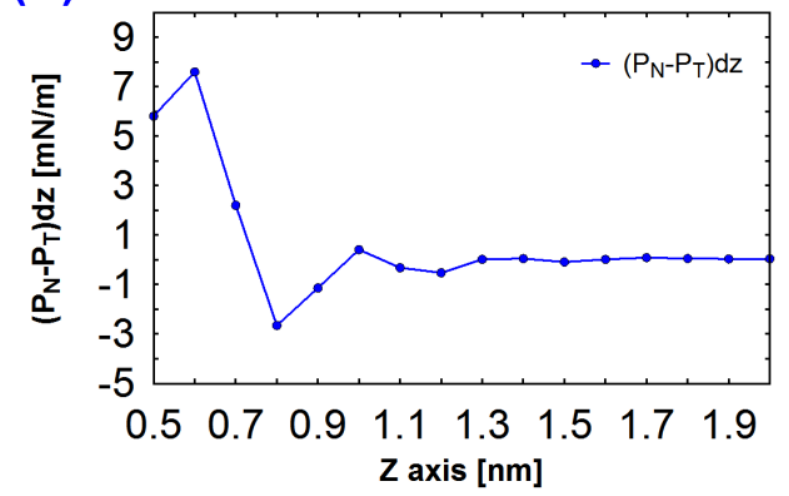

Figure S3. Average pressure, normal pressure and tangential pressure above platinum surface. (a) Normal and tangential pressure variation above surface up to $4 \mathrm{~nm}$. This shows a negative tangential pressure exists near surface and in monolayer the normal pressure is lower than tangential pressure. (b) Average pressure variation above the surface, which shows a peak positive pressure at the monolayer and strong negative pressures near the surface. (c) Difference between normal and tangential pressure components and its variation along the $\mathrm{Z}$-axis. This shows a strong shear stress at the monolayer. (d) Local surface tension variation along the Z-axis. 

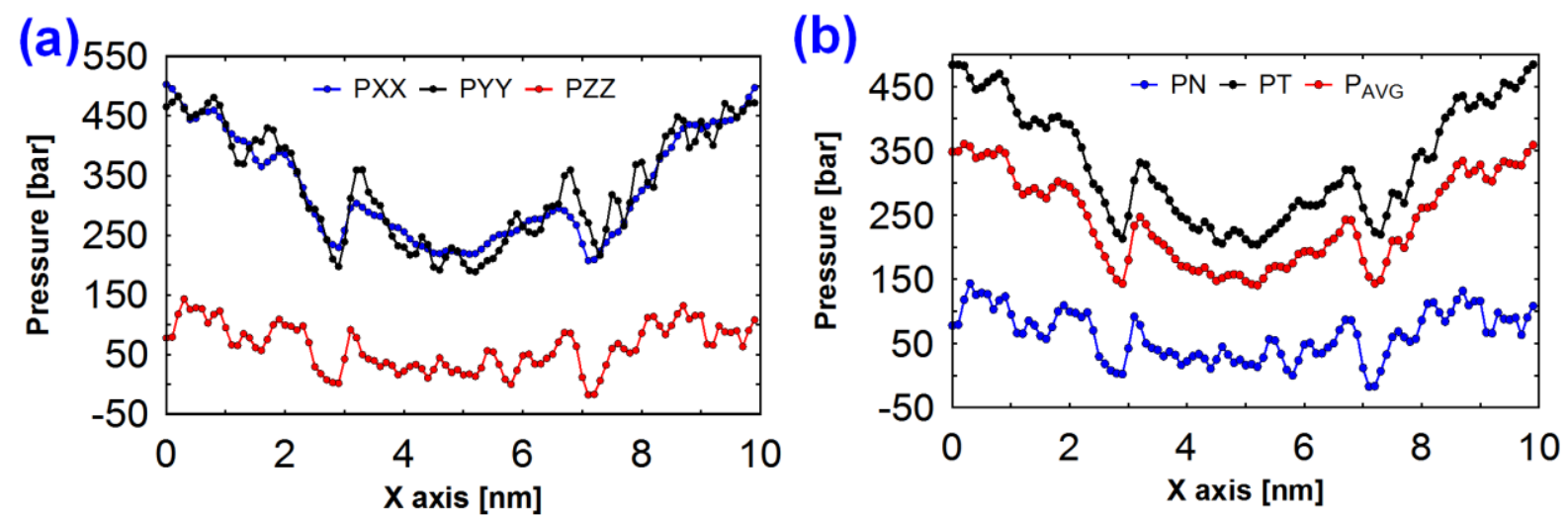

(c)

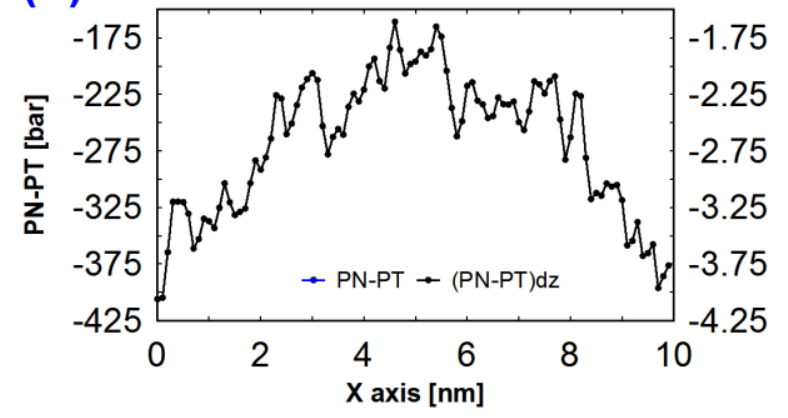

(d)

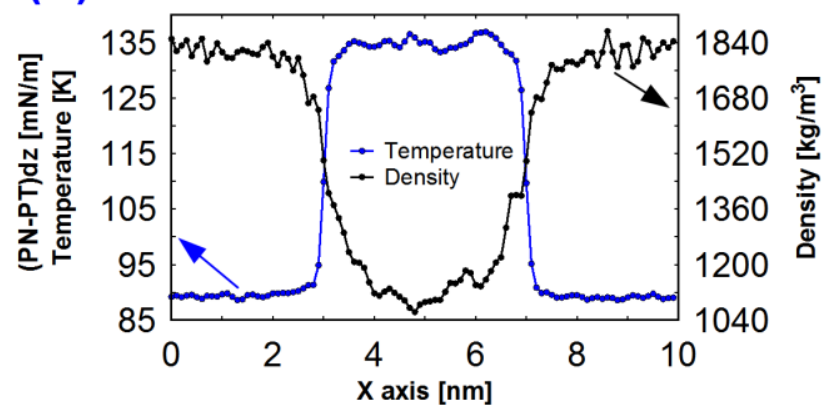

Figure S4. Monolayer pressure and surface tension estimation for $145 \mathrm{~K}$ middle heat case. (a) Pressure tensors $\mathrm{X}, \mathrm{Y}$ and $\mathrm{Z}$ variation along $\mathrm{X}$ axis in the monolayer. $\mathrm{X}$ and $\mathrm{Y}$ components (tangential) shows a steep decrease in the pressure towards the hot region, while $\mathrm{Z}$ component is not changed significantly. (b) Normal, tangential and average pressure variation in the monolayer. The average pressure also shows a trend of decreasing towards the hot region. (c) Surface tension variation along the monolayer. The left side axis of the graph shows the difference between normal and tangential components, the right side shows the local surface tension variation in $\mathrm{mN} / \mathrm{m}$. This plot shows a high surface tension near to the hot region which pulls the liquid from the colder region. (d) Density and temperature variation in the monolayer. The density shows a steep decrement towards the hot region. Temperature profile shows the hot region and cold regions.

\section{2D VELOCITY PLOTS}

The velocities of the atoms are smeared into an Eulerian 2D grid and temporal averaging is performed. This process is done for all the cases where the heating temperature is varied from 100 $\mathrm{K}$ to $150 \mathrm{~K}$, while keeping the end temperatures as $90 \mathrm{~K}$. The $2 \mathrm{D}$ velocity plots are shown in the Figure S 5 a-f. 
(a)

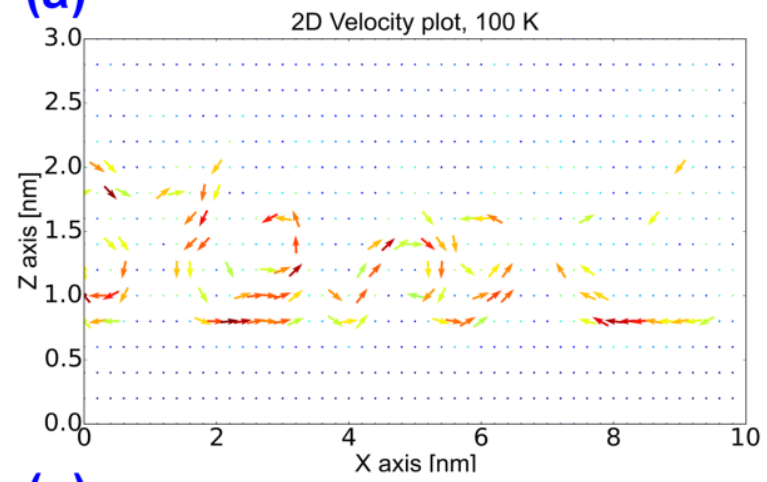

(c)

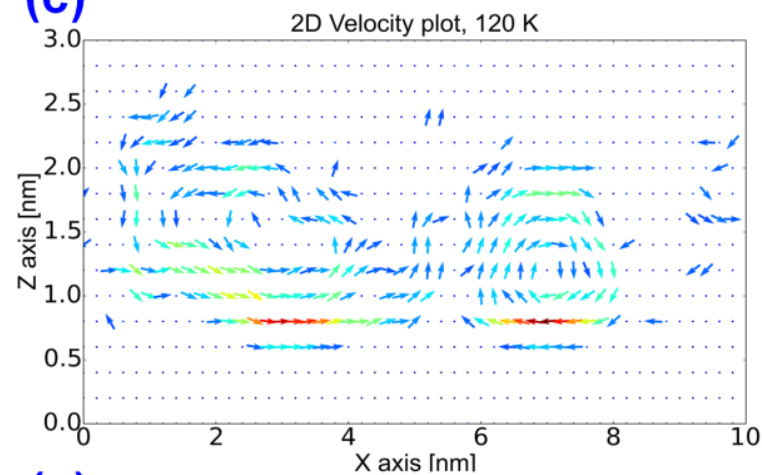

(e)

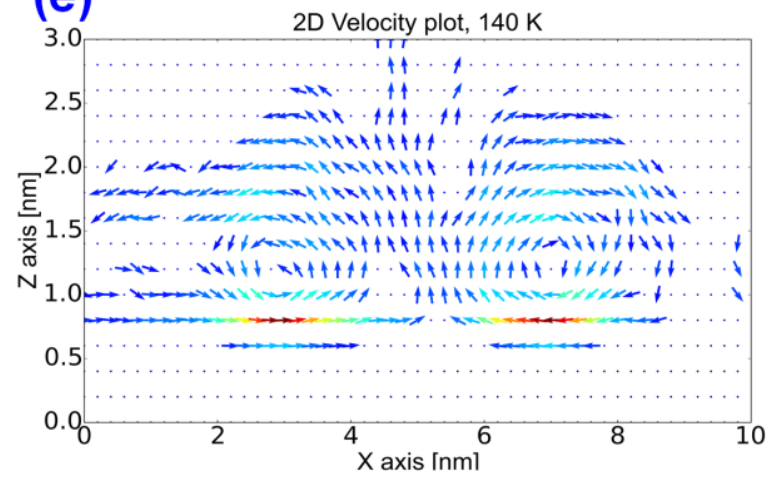

(b)

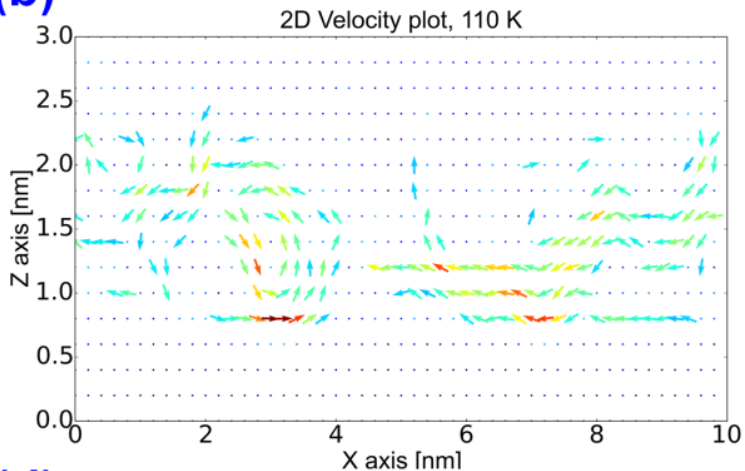

(d)

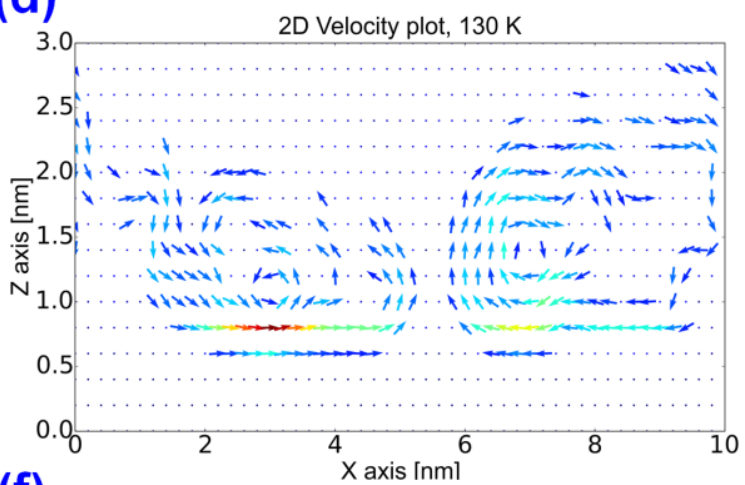

(f)

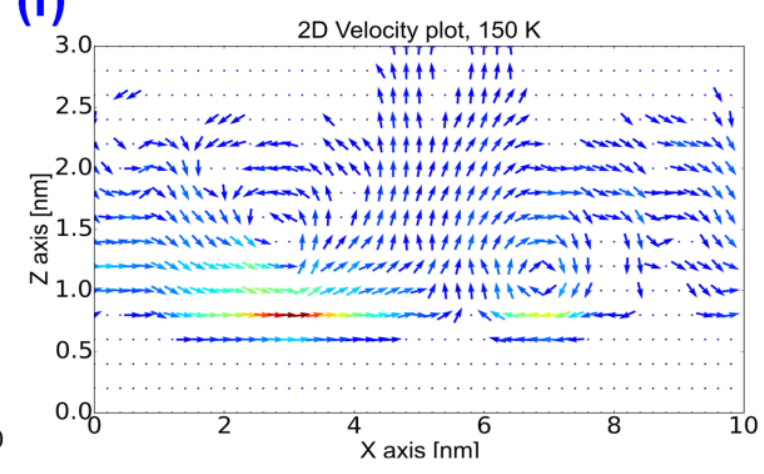

Figure S 5. (a-f) Velocity plots at varying heated region temperature from $100 \mathrm{~K}$ to $150 \mathrm{~K}$. The temperature of the cold sides remain at $90 \mathrm{~K}$ throughout the simulation. The arrows indicate the direction of flow and color indicates the strength of the flow (red means strong and blue means weak). The cases in which temperature gradient of the hot and cold side was beyond $50 \mathrm{~K}$ show a strong passive flow and steady evaporation from the surface.

\section{2D DENSITY PLOTS}

The density of the system is found using our modified Hardy's 2D interpolation scheme and assigned to a 2D grid. This temporal averaged quantity is shown in Figure S6a-f for different heating region temperatures. The thinning of monolayer (dense layer next to the wall) for higher 
temperature cases can be observed. Also the density of layer formation due to the influence of surface can also be seen here.

(a)

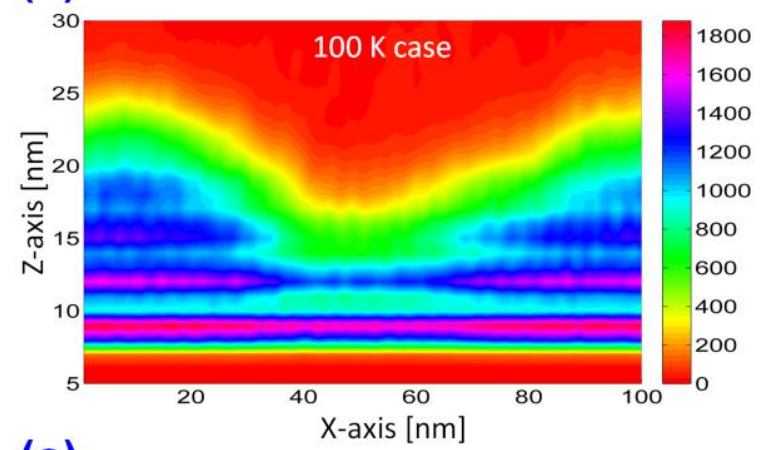

(c)

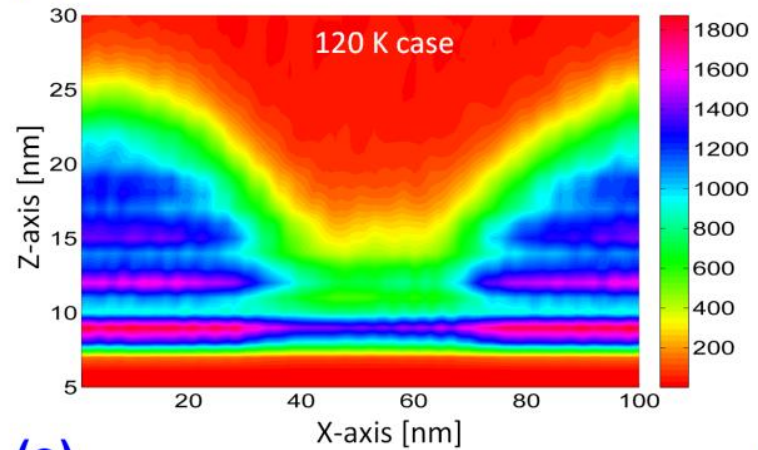

(e)

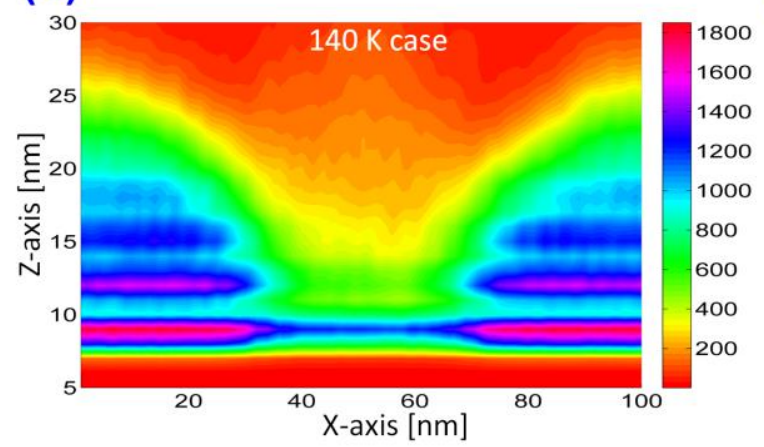

(b)

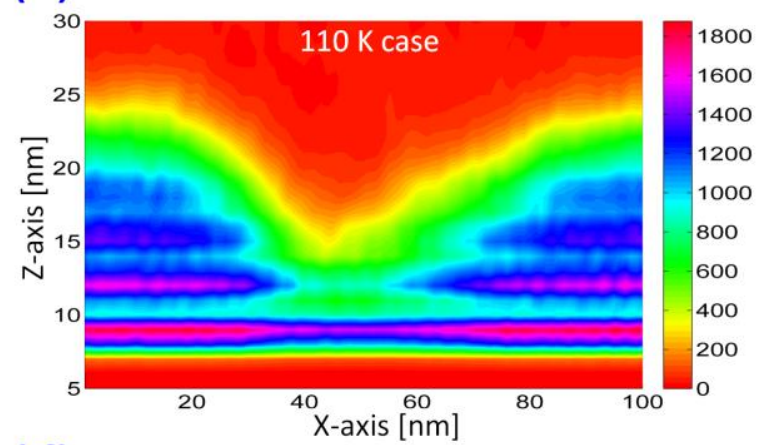

(d)

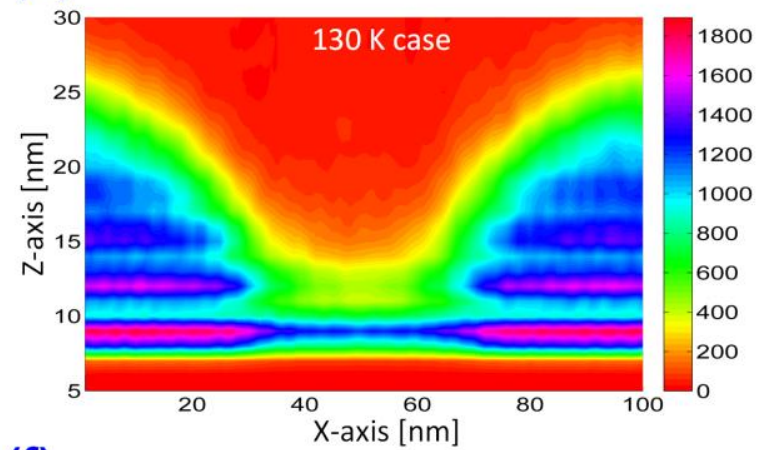

(f)

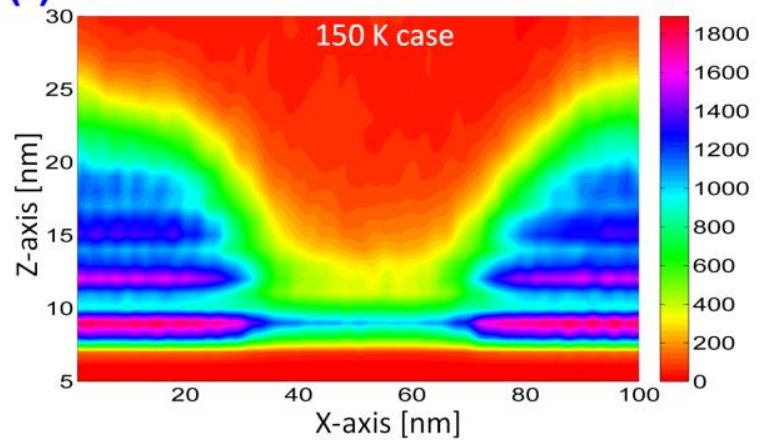

Figure S6. Density contour for different temperature cases. (a-f) Density contour of the 2D system is shown for various cases when mid temperature varies from $100 \mathrm{~K}$ to $150 \mathrm{~K}$. The red color shows the least dense regions and pink color shows the high dense regions. The density in the middle region changes significantly for the high thermal gradient cases (d,e,f) compared to the lower ones (a,b,c). This density reduction in the middle region is due to the evaporation of the liquid. Also we can see a thin layer of liquid is always present near to the surface and shows there are no dry-out situations, which is highly desirable requirement for a heat transfer device. 


\section{CONTROL VOLUME ANALYSIS FOR MASS FLOW ESTIMATION}

A control volume analysis is performed to estimate the mass flow rate of evaporation in the system. The control volume is selected based on the judgment about the most probable location of evaporation without immediate condensation of the argon. The mass flow rate is estimated by averaging the net velocity outflow and average density in the top portion of the control volume. This analysis is performed for different heating temperature cases ranging from $100 \mathrm{~K}$ to $150 \mathrm{~K}$ and shown in the Figure S7 a-f.

(a)
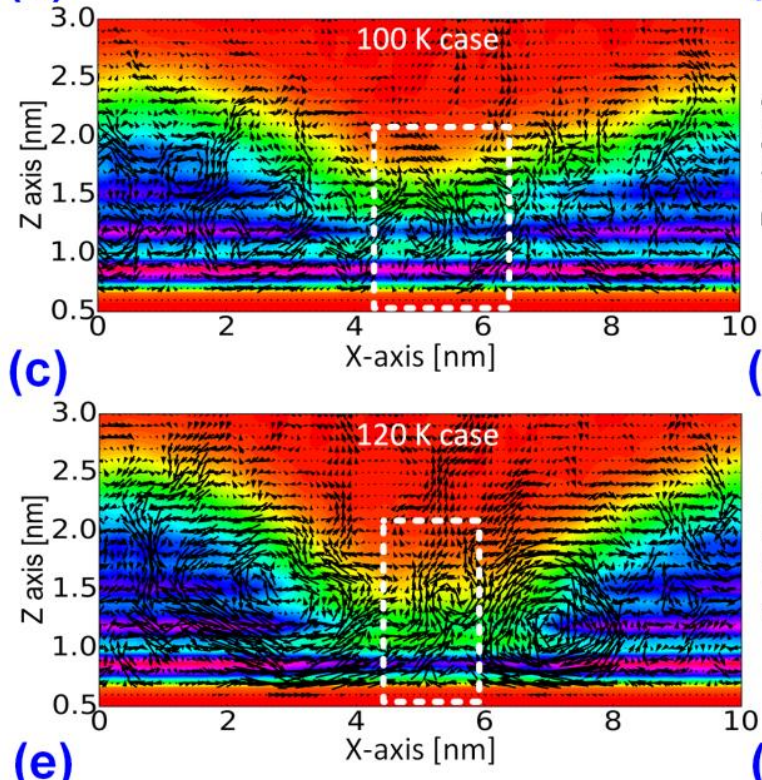

(e)

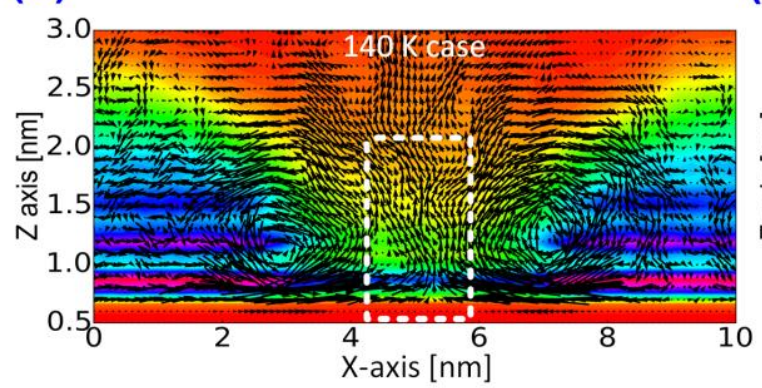

(b)

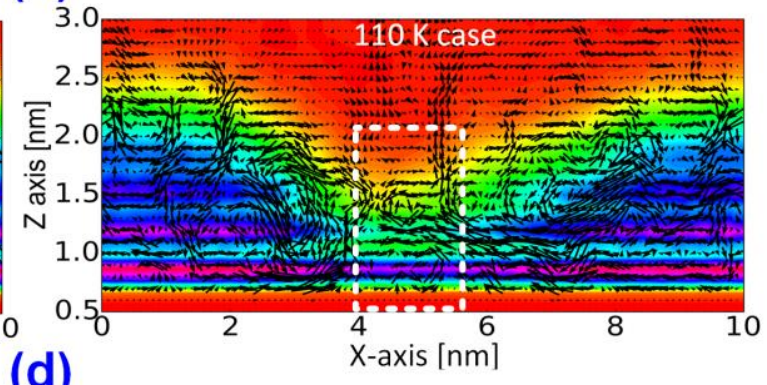

(d)

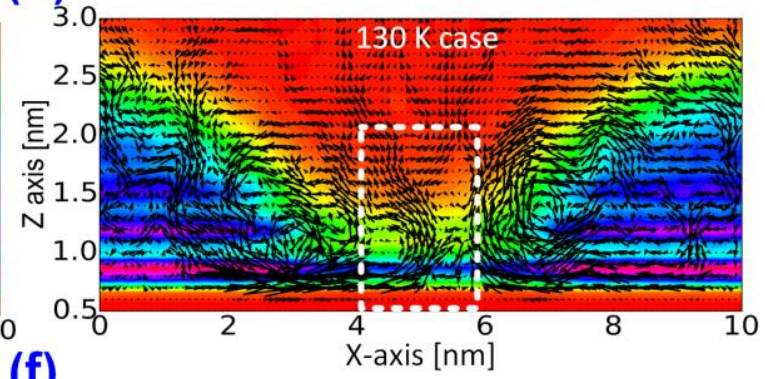

(f)

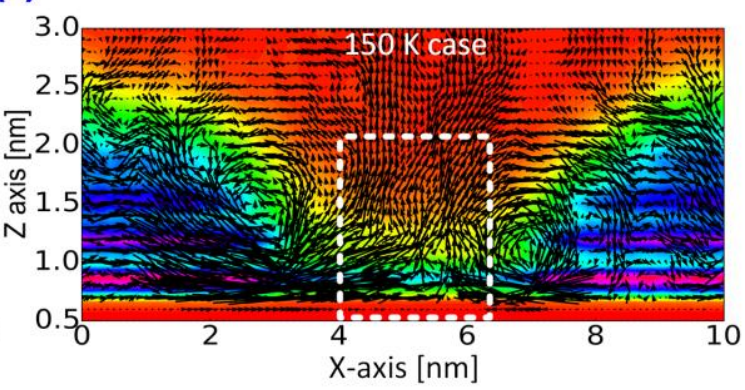

Figure S7. Control volume analysis at different heating temperatures. (a-f) Control volume defined based on the judgment of most probable location of evaporation is shown for the various cases. The panels here show the superimposed view of velocity profile on density contour. The selected control volumes, marked in white is used to determine the evaporation mass flow rate from the upper boundary, based on the density and velocity information. 